\title{
Mathematical Model of the Spatial Distributing of Density of Erosive Power of Multibubble Cavitation
}

\author{
Sergey Shestakov \\ Moscow state university of Technologies and Management \\ Russian Acoustical Society, Russia \\ E-mail: sdsh@mail.ru \\ Viktor Babak \\ JV "InvestGroup-White Russia", Republic of Belarus \\ E-mail: ig-belayarus@tut.by
}

Received: October 27, 2011

Accepted: November 23, $2011 \quad$ Published: February 1, 2012

doi:10.5539/apr.v4n1p64

URL: http://dx.doi.org/10.5539/apr.v4n1p64

\begin{abstract}
The research described in this paper shows that main parameter of applied researches of acoustic cavitation are not the intensity the temperatures of plasma in the cavitation bubbles (power of sonoluminescence), but the power of pressure pulses, which they produce, and which cause destruction of phases existing in a liquid (power of erosion). The distribution of the density power of erosion in space can be the subject of numerically simulated, if it is assumed that process of multibubble cavitation is an ergodic process. For this the integral of pressure superposition from all bubbles of cavitation field at any point in space, must be approximated by the function of the pressure pulse on the surface of a single cavitation bubble, that pulsate with a period equal to the period of oscillations of the harmonic wave. This superposition can be described using a two metrics of space, which belongs to this point. The first - the average distance from this point until all points of the cavitation region, determines the average time of arrival at this point of the total perturbation of pressure. Second - means harmonic distance and determines the average coefficient of attenuation of this perturbation. The results of computational and laboratory experiments illustrate the adequacy and the applicability of model. The model makes it possible to quantitatively compare the results of physical and chemical effects of cavitation in the any liquids in the reactors of any size and design. The model also gives a sufficient degree of accuracy and reliability of performing the technical calculations for the design of such devices and the possibility to make comparative assessments of the different reactors.
\end{abstract}

Keywords: Acoustic cavitations, Single-bubble and multibubble cavitations, Density of erosive power of cavitation, Mathematical model, Cavitational zone, Ergodic process

\section{Introduction}

Acoustic cavitation, which is caused by the elastic wave in the liquid, ripples the vapor-gas bubbles that concentrated under the action of the ponderomotive and hydrodynamic forces (Bjerknes \& Bernoulli) in the so-called cavitation fields near the nodes of sound pressure, where its amplitude exceeds a certain threshold $\mathrm{P}_{\mathrm{t}}$ (Mettin et al., 1997; Mettin, Koch \& Lauterborn, 2006; Shestakov, 2001a, 2009e). They produce the cavitation noise (secondary sound). This sound has the peak values of pressure and particle velocity, which are explained by the fact that waves from the bubbles take the form of shock waves due to the inertia of a dissipative dynamic system "bubble - liquid". The inertia is explained by transform the energy of the primary wave under the action the law of conservation of momentum of pressure (Shestakov, 2001a, 2009e).

Parameter of cavitation, by which determine the effectiveness of erosion and sonochemical action (its main physical and chemical phenomena, which are using at practical applications of cavitation) it is the pressure, what was achieved on the surface of bubbles (Margulis, 1995a), when they are compressed to minimum volume (collapse). Direct measurements of the collapse parameters are complicated by the requirement of expensive 
special hardware and are accompanied by methodical errors that arise when are used a majority of existing today for this methods and measurement tools. With this relate the ambiguity of mechanisms concepts of liquid-phase reactions outside of the bubbles and of gas-phase in them, as well as and accompanying these reactions the physical phenomena. For example, in respect of the light emission quanta, which accompanies pyrolysis of vapor-gas mixture under the compression of the bubbles, called sonoluminescence, there are very unequal opinions. And this despite the fact that along with the products of plasma-chemical reactions, through the spectrum of sonoluminescence do conclusions about the dynamics of cavitation processes (Margulis, 1995a). By the thermal radiation explain the sonoluminescence of the multibubble cavitation rarely (Dezhkunov et al., 2000a), more often - of the single-bubble cavitation (Margulis, 2000c), although and in it also find differences from the spectrum of a hot body (Matula, et al., 1995; Flannigan \& Suslik, 2005). The sonoluminescence has no known practical applications, though, if it is a radiation of hot body, by virtue of the laws of Wien, Planck and Stefan-Boltzmann, she is associated with the pressure on the bubbles surface and can be used to estimate the intensity of cavitation. True, there are proposals to use her as an X-ray source (Shestakov, 2008d) or for obtaining a controlled periodical thermonuclear reaction (Flynn, 1982; Putterman et al., 1994; Margulis, 1997b; Taleyarkhan et al., 2002, 2004; Nigmatulin, 2005; Lahey, Taleyarkhan \& Nigmatulin, 2007; Khavroshkin, Bystrov, 2007; Shestakov, 2007b).

In applied research directed to the cavitation effects, mainly of cavitation erosion of solid surfaces, use the different methods of measuring and analysis of the spectra of secondary sound (Shestakov, 2007b; Dezhkunov, Ignatenko \& Kotukhov, 2007; Lanin, Dezhkunov \& Tomal, 2008), as well as simple and clear method of evaluating the cavitation damage of metal samples, for example, of aluminum foil (Krefting, Mettin \& Lauterborn, 2004). And this is directly related to sonochemistry, since many from its applications, such as food sonochemistry (Shestakov, Krasulya, 2010) are based on sonochemical reactions of the dehydration in the aqueous solutions that is epithermal destruction of hydrogen bonds between water molecules, the ions and molecules of the substances dissolved in water (Rogov, Shestakov, 2004). This reaction is not accompanied by dissociation of molecules into the ions and for this is enough energy of cavitation erosion, which causes destruction of substance at the molecular level by changing the dipole-dipole and ion-dipole interactions in this substance (Ashokkumar, Rink, Shestakov, 2011). It is known that water even at room temperature has a molecular structure similar to structure of ice, which too is formed by hydrogen bonds (Jinesh, Frenken, 2008). Therefore at water there is a reaction, which by epithermal way can be caused by the cavitation (Shestakov, Krasulya, 2010):

$$
n \mathrm{H}_{2} \mathrm{O} \rightleftarrows\left(\mathrm{H}_{2} \mathrm{O}\right)_{n}+Q
$$

where $Q$ - heat of hydration or energy of dehydration.

Thus, the phenomenon of acoustic cavitation sufficiently is studied and described only at the level of phenomenology, empiricism and the methods of investigating the possibilities of its practical application, which base on laboratory experiments and physical modeling of processes and devices. These experiments because of the ambiguity of representations of the knowledge in this area are low interpretability and reproducibility of results, and it is difficult of the physical model "to increase scale" (Mawson, Knoerzer, 2007) at creation of industrial device. Possession of the mathematical model of the cavitation and the principles of numerical comparison between processes of cavitation allows to develop applied sonochemistry and equipment without labor-intensive stages of creating a working model of industrial sonoreactors and to rely on the results of laboratory optimization of a process. Formal description of cavitation allows to reduce the scope of research and to perform them at the expense of laboratory experiment and creation of necessary technology and equipment through a numerical simulation, based on its results. While likely absolutely to refuse from experimentation will be impossible, but it will be a laboratory process which make with small amounts of ingredients and with the small-sized technique (Shestakov, 2010f).

\section{Model of Single-bubble Cavitation and the Computational Experiments with Her}

Theoretical aspects of cavitation and the computational experiments in this field are based on the differential equations of motion the wall of spherical vapor-gas cavity under the influence of an alternating external pressure (Knapp, Daily \& Hammitt, 1970; Rozenberg, 1968). Analysis of variants of known equations of this kind was carried out repeatedly by different researchers. For example, one of equations belongs to J.W. Strutt (Lord Rayleigh). It is also amply fulfilled in the book by R. Knapp, J. Daily and F. Hammitt. Of the equations, which most completely take into account the physical aspects the phenomenon of the cavitation, can make the mathematical model, fit for numerical analysis of the parameters of pulsating single cavitation bubble. For example, the current radius size of a wall of bubble $R$ and current pressure $P$ upon a wall, if the bubble is filled 
by a mix of a gas and a steam of liquid with an adiabatic curve indicator $\gamma$, the its initial diameter $2 R_{0}$ and he pulsate under action of sinusoidal changing pressure with amplitude $A$ and frequency $f$ in a liquid in density $\rho$ with viscosity $\mu$ and with factor of a superficial tension $\sigma$, it is possible to describe, by integrating system of the equations with initial conditions $R=R_{0}, \dot{R}=0$ :

$$
\left\{\begin{array}{l}
\ddot{R}=\frac{H}{R}\left(\frac{C+\dot{R}}{C-\dot{R}}\right)-\frac{\dot{R}^{2}}{2 R}\left(\frac{3 C-\dot{R}}{C-\dot{R}}\right)+\frac{\dot{H}}{C} \\
P=\left(\frac{R_{0}}{R}\right)^{3 \gamma}\left(P_{h}-P_{v}+2 \frac{\sigma}{R_{0}}+4 \mu \frac{\dot{R}}{R}\right)
\end{array}\right.
$$

where: $\quad C=\left(\frac{n}{\rho}\right)^{\frac{1}{2}}(P+B)^{\frac{n-1}{2 n}}\left(P_{h}-A \sin 2 \pi f t+B\right)^{\frac{1}{2 n}}-$ speed of sound in the liquid at the bubble wall; $H=\frac{n}{\rho(n-1)} \cdot\left(P_{h}-A \sin 2 \pi f t+B\right)^{\frac{1}{n}}\left[(P+B)^{\frac{n-1}{n}}-\left(P_{h}-A \sin 2 \pi f t+B\right)^{\frac{n-1}{n}}\right]-$ the enthalpy on the bubble wall; $\dot{H}=-3 \gamma P \frac{\dot{R}}{R}[n(P+B)]^{-\frac{1}{n}}$ - derivative from an enthalpy on the bubble wall; $P_{h}, P_{v}$ and $B, n-$ the hydrostatic pressure in the fluid, the partial pressure of vapor and the parameters of the equation of her estate; $t$-dimensionless time in fractions of a period of a harmonic wave $T$.

On Fig. 1 and 2, are shown the results of computational experiments with a model of single bubble, namely, in Fig. 1 - which obtained by numerical decision (2) in the sets of points (size $6 \cdot 10^{4}$ ) for three randomly, selected periods of the harmonic wave: a). Logarithms of the dimensionless pressure on bubble surface $\lg p=\lg \frac{P}{P^{*}}$, where $P^{*}$ - pressure at a time when the instantaneous value of its diameter is equal to the initial value (10 $\mu \mathrm{m}$ ), which pulsate under the influence of the elastic harmonic wave with frequency of $20 \mathrm{kHz}$, and with four different sizes of amplitude of the sound pressure in the chemically pure water with hydrostatic pressure equal to atmospheric; b). dimensionless pressure impulses $I=\int_{0}^{3 T} \frac{P-P^{*}}{P^{*}} \mathrm{~d} t-$ the integral characteristic, in which is included

both pressure and time of its appendix (Podobriy et al., 1969); c). Phase trajectories on a phase plane "impulse of pressure - the logarithm of pressure" of change of the parameters of dynamical system of the bubble.On Fig. 2 in the same format are shown the results of calculation of parameters of a pulsation in water of bubbles with various diameters and amplitude of sound pressure $A=5$ bar.

\section{Discussion Computational Experiments with Single-bubble Model}

It should be noted that the influence of viscosity of liquid on the process of pulsation of cavitation bubbles in her are still not completely the deterministic parameter of model and along with the influence on the dynamics of process such factors as a possible vapor condensation in the bubble, heat and mass transfer through its walls and the violation of his spherical symmetry (Klotz., Hynynen, 2010; Melnikov, Makarenko \& Makarenko, 2004; Gaitan, Tessien \& Hiller, 2007), may reduce the adequacy of the model. The pressure impulses on surface of a cavitation bubble during depression and compression in accordance with the law of conservation of energy, on average over an infinitely long time must be equal in magnitude (as well as on the front surface of wave of an underwater explosion (Podobriy et al., 1969). Otherwise, the average value over the period of oscillation of pressure in the fluid will be to infinitely increase or to fall. This will lead to spontaneous cessation of cavitation due to the complete removals the gas from the fluid, what in reality is not observed. On the contrary, experience shows that the acoustic cavitation in case remove heat, which is formed by the dissipation of energy of the elastic vibrations on the internal friction in the liquid possesses self-organizing and seeks the balance, which belongs to liquid with any amplitude of sound pressure. Therefore, in experiments the size $P^{*}-$ is an arbitrary parameter, and is different from the initial pressure in the bubble $P_{0}$, so that his mathematical expression include also a resistance to movement of its wall, what creates a viscosity of liquid. In this case it's chosen such that phase trajectory in the phase plane (Fig. 1c, Fig. 2c) would have the form of limiting cycles. The experiments were executed at different sizes of the input parameters of the acoustic plane wave in the water, above which they are unlikely to be used in real conditions. For example, the maximum amplitude of sound pressure matched for intensity ultrasound $70 \mathrm{~W} / \mathrm{cm}^{2}$. Under these conditions $P^{*}$ as well as the maximum speed of compression of a bubble, depends from $A$ and is in limits $P_{0}<P^{*}<A$. 
Analysis of the graphs on Fig. 2 shows that with increasing A, the maximum value of $p$ has increase and, obviously, reaches the limit of growth near the collapse of bubble, which occurs at the end of half-period of compression of the harmonic wave. With further increase $\mathrm{A}$ at the conservation of an equal numbers of collapses and numbers of periods of the wave k, p on the average becomes equal to this limit (see Fig. 1a,c and Fig. 2c). The diameter of the bubble near equality of the periods of pulsation of the bubble and harmonic wave has only little influence on the form of the pressure impulse (Fig. 2b).

Unfortunately, using this single-bubble model can not describe field consisting of a set of a many bubbles, because she does not take into account the presence of the force field of the secondary sound. Over a preparation of such equations for the whole cavitation field in the middle of last century worked the famous Russian explorer of cavitation - Professor L. Rosenberg (Acoustics Institute of the Academy of Sciences USSR), He wrote: "... even the formation of these equations - the question of enormous difficulty, not to mention their integration. Therefore, the solution of the problem in the future (Rozenberg, 1968)». But if to remember about the ergodic theory (what through the description of behavior in time of one of the elements of the force field describes variation of all this force field in the geometrical space) then, as we did, separately is consider a single bubble, which is part of the cavitation region and it greatly facilitate task.

Analysis of single-bubble model allows us to make an important conclusion for modeling multibubble cavitation: The limit cycle of the bubble dynamics especially near his pulsations in equal periods with a harmonic wave - are stable (see Fig. 2c). And cavitation of the ensemble, consisting of a set of bubbles, which is managed by external pressure of the sound, can be considered as ergodic dynamic system. This, as will be shown below, allows us to consider the distribution in space of the instantaneous parameters of the cavitation field through a dynamics of single bubble in the time and paves the way for the equations of multibubble cavitation region.

\section{Ergodic Model of Multibubble Cavitation and Computational Experiments with Her}

In studies carried out under the direction of Professor Rosenberg in 1950 - 1960 was formulated the generalized approach to the estimation of energy of the elastic waves, which is dissipated on a multibubble cavitation, based on empirical data. But attempts of formalized description of the cavitation fields, as functions of the spatial distributions of energy, which transformed through cavitation, began to emerge only recently (Mettin, Koch \& Lauterborn, 2006; Shestakov S. 2007c, 2008d, 2009e; Gaitan, Tessien \& Hiller, 2007; Lavrinenko, Savina. \& Leonov, 2007). One of them make for the cavitation region, which acts in a standing wave with one antinode of pressure, led to the following considerations, which dictate significant changes to improve this description.

It is known that a cavitation erosion decreases inversely to the square of the distance from the destruction object to a cavitation bubble, while the attenuation of pressure fluctuations - approximately inversely to this distance [29, 30]. That is, for the destruction the secondary sound does work on the object. Measure of work is size of energy of the destruction, located in the each physical point of surface. Being divided into the duration of action, she can be named $q_{\mathrm{er}}$ - the volumetric density of power of cavitation erosion (VDPCE) at this point. Thus, the problem of modeling the dynamics of the cavitation field is reduced to finding the distribution of qer in the coordinate space $(x, y, z)$, with the beginning of coordinates, for example, is coincide with the geometric center of the subspace $\mathrm{v}$ (or of the $i$ volumes $\Sigma v_{i}$ ), which occupies a pulsating bubbles. To the each point of space the cavitating liquid belong the finite number of straight line pieces (vectors $\mathbf{r}$ ), which connect this point with all $\mathrm{N}$ bubbles. If all the bubbles have the same dimensions, then they cause instantaneous fluctuations of pressure anywhere in the cavitation region of one harmonic half-wave, which looks like on:

$$
\Delta P=\frac{N}{v t} \iiint_{t} \int_{(v)} \frac{\left(P-P^{*}\right) R_{0}}{R_{0}+r} \mathrm{~d} x \mathrm{~d} y \mathrm{~d} z \mathrm{~d} \tau,
$$

where: $r$ - modulus of the vector $\mathbf{r} ; t$ - mean dimensionless arrival time of pressure fluctuations from all cavitation bubbles to the selected point.

Instantaneous value of the deviations of the pressure $P-P^{*}$ on the surface one of bubbles on the average over on big sizes of time in (2), is under the signs of the two integrals (by the $t$ and $v$ ). This is done, because the $P-P^{*}$ depends on the phase of the total pressure fluctuations fluctuations, which in turn depends on the linear sizes of the cavitation field. If the bubbles pulsate with periods that coincide with periods of a harmonic wave (see Fig. 2), and the dimensionless pressure impulse on the surface of a single bubble is phase function, it means that a dimensionless phase of this pulsations can be used to find the average phase of the superposition of pulsations on the geometric space of cavitation field at a given point. Then, dropping in (2), having removed $R_{0}$ because of its smallness in the integrand, and dividing the variables, we may write the form of function for mean square fluctuation of the pressure from cavitation for period of the wave at any point of space of the liquid: 


$$
\overline{\Delta P^{2}}=\left(\lambda P^{*} R_{0} N \frac{\iiint_{(v)} r^{-1} \mathrm{~d} x \mathrm{~d} y \mathrm{~d} z}{\iiint_{(v)} r \mathrm{~d} x \mathrm{~d} y \mathrm{~d} z}\right)^{2} \int_{0}^{1} I_{p}^{2} \mathrm{~d} \phi,
$$

where: $\lambda$ - the length of the harmonic wave, equal to wave length of the pressure fluctuations from cavitation; $\phi$ - the dimensionless phase of the harmonic wave. The expression (4) can be simplified by introduction the special metrics of space. That $\overline{\mathrm{m}}_{a}=v^{-1} \iiint_{(v)} r \mathrm{~d} x \mathrm{~d} y \mathrm{~d} z \quad$ and $\quad \overline{\mathrm{m}}_{h}=v\left(\iiint_{(v)} r^{-1} \mathrm{~d} x \mathrm{~d} y \mathrm{~d} z\right)^{-1}$ - average arithmetical and averageharmonical distances from a given point to the all points of the cavitation region, respectively. The first, after dividing on the length of wave $\lambda$ becomes the average time of arrival of pressure fluctuation from the all bubbles of the region of cavitation to the point, for which make calculation $t=\frac{\overline{\mathrm{m}}_{a}}{\lambda}$. By dividing the initial radius of abubble on the second metric of space, we obtain $a=\frac{R_{0}}{\overline{\mathrm{m}}_{h}}$ - attenuation coefficient of the total fluctuation of ressure from all cavitation bubbles in this point.

Length of free run of the pressure fluctuation from cavitation during the period of the harmonic wave in (4) is equal to the length of this wave. This can raise the doubts as to the correctness of the interpretation (3) in connection with the famous hypothesis of Kirkwood and Bethe (Knapp, Daily \& Hammitt, 1970; Rozenberg, 1968; Lavrinenko, Savina \& Leonov, 2007; Kedrinskiy, 1975), which eliminates by Fig. 3. On Fig. 3 shown that the deviation of the absolute sound velocity near the bubble surface from the nominal occur very quickly and has an alternating sign. Average speed of moving into liquids of impulses of pressure from the cavitation bubbles during their pulsation, which is exceeding duration of a collapse almost in 50 times, consisting from the speed of a sound and speed of movement of a wall, remains equal to rated speed of a sound in liquid.

For an approximating the function of the pressure impulse, equal on time to the period of harmonic wave in (Shestakov, 2008d) am offere to use piecewise linear function $I=[t+\phi]-t$, where (and further) in square brackets - is the whole part of number. Derivative of this function is the periodic generalized function of two real dimensionless variables, which in each $k^{\text {th }}$ period can be expressed through $\delta$-Dirac:

$$
\dot{I}=\delta-1=\left\{\begin{array}{l}
\infty, \text { if } t=T_{k}-\phi \\
-1, \text { if } t \neq T_{k}-\phi
\end{array}\right.
$$

Such reception is similar to the receptions applied in the quantum mechanics where for the description of systems with the concentrated parameters also are used the generalized functions, their derivatives and integrals. If differentiation of function of an impulse of pressure gives as a result function of a variable component of pressure upon surfaces of a bubble with final jump in a collapse point, derivative from $I_{p}$ there has a point of rupture of $2^{\text {-nd }}$ sort. Time of stay of a bubble in volume smaller than equilibrium volume in relation to the period of a harmonious wave is very small (see Fig. 3). Therefore, such approximation doesn't obstacle to authentic description of total impulse of pressure from cavitation area and integration on phase its square with formation as a result of the simple expression of a kind $\{t\}-\{t\}^{2}$, where (and further) in the figured brackets - is the fractional part of number. As a result of the made transformations is possible to rewrite (3) for a cavitation in antinode of pressure in one standing half wave, as:

$$
\overline{\Delta P^{2}}=\left(D v P^{*} a\right)^{2} \frac{\{t\}-\{t\}^{2}}{t^{2}},
$$

where $\mathrm{D}$ - conditional volume density of the bubbles in liquid.

In practice, we often have to deal with cavitation in the wave, which contains $\mathrm{n}$ antinodes of pressure and, respectively, $\mathrm{n}$ of the areas of cavitation. In modeling thus there are two problems. Solution one of them, which consists in formalization of the energy dispersions on cavitation of acoustic vibrations in the plane wave is offered for calculation of cavitation reactors in the (Shestakov, Befus, 2008). There the sizes of each volume $v_{i}(i=1,2 \ldots$ $n$ ) determine by the square of wave front and by heights $i^{- \text {th }}$ of cavitation regions on her beam. Dependence of the modulus of resulting amplitude of the sound pressure in the linear approximation is expressed through $\eta-$ coefficient of dispersions of wave energy on the cavitation (Rozenberg, 1968), $\theta$ - coefficient of wave reflection and $A_{0}$ - amplitude of sound pressure on the surface of radiations of a ultrasound into the half-space of the liquid: 


$$
A=A_{0}|\sin 2 \pi f t| \cdot\left\{\begin{array}{l}
\left(1-\eta^{\frac{1}{2}}\right)^{2 \frac{\phi}{\pi}}+\left(1-\eta^{\frac{1}{2}}\right)^{4 n-2 \frac{\phi}{\pi}} \theta \\
\left(1-\eta^{\frac{1}{2}}\right)^{2 \frac{\phi}{\pi}}+\left(1-\eta^{\frac{1}{2}}\right)^{2 n-2 \frac{\phi}{\pi}}
\end{array}\right.
$$

at one and two identical sources of coherent waves, which are directed towards each other, respectively. The coordinates of the boundaries of volumes vi on the beam of wave in angular units calculated as positive roots of the transcendental equation $A-P_{t}=0$, and their heights hi on the beam of are defined as the difference between odd and even his roots. Their converting to linear unit doing by dividing on the wave number $\frac{2 \pi}{\lambda}$.

The next problem - this a fact what the cavitation fields the located next to each other on beam of wave, is have the opposite phase of pulsations. It is solved by the introducing the correction to $t$, which depends on $n$ through $\overline{\mathrm{m}}_{a}$. For example, for even antinodes of pressure the amendment to $t$ will be equal $\frac{\lambda}{2}$. From here $t+\frac{1}{2}=t^{*}$, and the decision for $q_{e r}$ it is a proportion of combinatory function (Ashokkumar, Rink \& Shestakov, 2011). For example, if $n=2$ :

$$
q_{e r} \sim f\left(v_{1}+v_{2}\right)^{2} a^{2}\left(\frac{\{t\}-\{t\}^{2}}{t^{2}}+\frac{\left\{t^{*}\right\}-\left\{t^{*}\right\}^{2}}{t^{*}}-\frac{2\{t\}\left\{t^{*}\right\}}{t^{*} t}+\frac{2}{t^{*} t}\left\{\begin{array}{ll}
\{t\} & \text { if }\{t *\}>\{t\} \\
\left\{t^{*}\right\} & \text { if }\left\{t^{*}\right\} \leq\{t\}
\end{array}\right)\right.
$$

Then, expressing $\int_{0}^{1} I^{2} \mathrm{~d} \phi$ at (4) as function of arguments $t, \lambda$ and $n$ for infinite number of wave periods, regardless of initial phase, VDPCE for specific fluid can be written in the following proportions:

$$
q_{e r} \sim f\left(\sum_{i=1}^{n} v_{i}\right)^{2} a^{2} \frac{\left\{t+\frac{1}{2 n}\left[\frac{n}{2}\right]\right\}-\left\{t+\frac{1}{2 n}\left[\frac{n}{2}\right]\right\}^{2}}{t^{2}+t \frac{1}{n}\left[\frac{n}{2}\right]+\frac{1}{4 n^{2}}\left[\frac{n}{2}\right]^{2}}
$$

In the wave with amplitude of sound pressure, at which the cycles of pulsations of the bubbles occur simultaneously with periods of wave, this function allows to calculate numerically the spatial distribution VDPCE irrespective of time. Two distribution of VDPCE for the period of the primary wave, calculated in accordance with (8) for its specific initial phase is on Fig. 4ab. One more that is averaged over an infinitely large time, in accordance with (9) are on Fig. 4c. Fig. 5a is shown the results of the evaluation the power of cavitation erosion, which are not included in the paper (Shestakov, 2008d), by the method described by R. Knapp more than half a century ago, but which frequently is used in recent studies (Krefting, Mettin \& Lauterborn, 2004). In this case, in a special reactor with the three sources of standing half-waves of identical frequency, phase and intensity with the parallel beams in the plane of the antinode of sound pressure was placed sample of aluminum foil, which was subjected there to cavitation erosion. General view and the scheme of the reactor, is shown in Fig. 6 (is taken from Shestakov, 2008d). Fig. 5b shows the distribution pattern of this VDPCE in a plane of foil the received at its computer simulation. Fig. 7a shows the results of an experiment of erosion of the aluminum foil, and Fig. $7 \mathrm{~b}$ the result of computer simulation of the distribution the relative VDPCE in this case. The sample has been established, as shown by dotted line, in parallel to a wave beam in volume of the laboratory reactor (Shestakov, Befus, 2008). In the reactor has been removed the basis with a reflector. Reactor has been inverted and filled by a water at level $68 \mathrm{~mm}$ above a surface of the emitter of the basic wave, Thus, this wave has been reflected from a water surface which has received as a result of it contact to air with very low acoustic resistance.

\section{Discussion of Results of the Computational Experiments with the Model of Multibubble Cavitation. The Hypothesis of the Similarity of Cavitation Processes}

In comparative experiments (Fig. 5) we utilized sources of ultrasound from the processor UIP-250 German-made with waveguides for the amplitude of sound pressure in the water 20 bar (Shestakov, (2008d), and only 2 bar (Shestakov, Krasulya, 2010) (Fig. 7). However, the damage of the aluminum foil caused by cavitation in three coherent half-waves, and also in the two areas of cavitation of one wave, are a clear demonstration of sufficient adequacy of the model, even though period of sound wave and periods of oscillation of cavitation bubbles can be and not coinciding. Comparison of this destruction of the foil with the distribution functions of the VDPCE shows that the model is applicable even in the presence of several independent waves in the liquid, if the 
oscillations are coherent. The phenomenological description of this model also can explain established, for example, in (Lahey, Taleyarkhan \& Nigmatulin, 2007; Mettin et al., 1997) fact of the dependence of the pressure achieved at the collapse of a bubble from the size of the all cavitation field.

The parameters $N$ or $D, R_{0}$ and $P^{*}$ - the constants, that characterize a specific liquid and its behavior at cavitation process. Therefore, if in some way become known and used in the calculation their sizes, obtained in absolute units for particular fluid, the numerical results of its can be applied only to this fluid. In practice, the study of any applications of cavitation usually starts with a practical process optimization in the laboratory.

For scale increases of laboratory sonotechnology to be suitable at industrial process first make the prototype of industrial equipment, or, using the similarity of cavitation processes on different sizes, make a numerical model of industrial technology, the results of which will be used for design the industrial devices, bypassing the stage of prototype. It is clear that the second option makes the project much cheaper, and the model described above allows implementing it.

With such strategy of design of equipment and technology a fluid in the research lab and in production is same, and we can use the model to select the optimal acoustic parameters and required sizes for the industrial reactors in absolute dimensions, knowing these characteristics of device, which was used for optimization in the laboratory, but without knowing absolute parameters of this liquid. For example, result of chemical process in the laboratory reactor with working volume $V_{\mathrm{lab}}$, which has some size of average VDPCE and its distribution in this volume, achieved the optimum. It can be assumed that in the reactor arbitrarily chosen volume, with other conditions of wave formation, but same dispersion of distribution VDPCE in the same liquid can be reached a productivity, which differs in

$$
\frac{f \iiint_{(V)} \overline{\Delta P^{2}} \mathrm{~d} x \mathrm{~d} y \mathrm{~d} z}{f_{\text {lab }} \iiint_{\left(V_{\text {lab }}\right)} \overline{\Delta P_{\text {lab }}^{2}} \mathrm{~d} x \mathrm{~d} y \mathrm{~d} z} \text { time. }
$$

The equal constants in this expression and units of dimensions of other variables in the numerator and denominator cancel each other and in result is obtained a dimensionless parameter - the ratio of productivity the reactor, which, for example, is project and of laboratory reactor, the productivity and other technical characteristics of which (the need for energy, the sizes, the weight etc.) are known. This makes it possible to compare them with each other in processing of liquids with same parameters. That is hypothesis of the similarity of cavitation processes. She has need to confirming.

\subsection{Experimental confirmation of the similarity hypothesis of cavitation processes}

The practical test of this method of comparison of the cavitation processes is carried out on well-known effect of dispersing by cavitation the phase of aqueous sols. As the experimental setup (Fig. 7) is used cavitation reactor with the casing of working volume, made of standard stainless seamless pipe Ø48 $\mathrm{mm}$, which has two channels for flow of sol. At the ends of this casing through O-rings are mounted two of acoustic transformers that transmit fluctuations of the primary sound from emitters to liquid. The distance between their emitting surfaces in the first case (Fig. 7a) is equal to $0,5 \lambda$, and in the second (Fig. 7b) $1,5 \lambda$. In the first version (Fig. 7a) a source primary sound with oscillation frequency of $22 \mathrm{kHz}$ was connected to one of the transformers (the other acted as a reflector). In the second version (Fig. 7b) each of transformers is attached to the source. Their waves are coherent.

Cavitation dispersing was subjected the sol of polystyrene latex, which was obtained by emulsion polymerization of styrene, with average diameter of sphere of latex particle $300 \mathrm{~nm}$. In him the cavitation erosion make work against the strength of the particles of polystyrene. That is proportional to change the dispersion variation of sol. In the experiment, five samples of latex with the equal initial optical density passed through the reactor with a rate of $2 \mathrm{ml} / \mathrm{s}$ at the equal initial temperature. Then, by a monochrome turbidimetry on the wavelength $400 \ldots 600$ $\mathrm{nm}$ with a resolution of $20 \mathrm{~nm}$ was determined their optical transmittance. Transverse sizes of the particles was calculated from the angles of slope of the graphs of the logarithms of optical density, assuming the form of particles the spherical and using the known empirical equation of Geller.

In calculating the relations of productivities of the reactors of the second and first version $P_{t}$ are set a 2 bar, as it approximately corresponds to the pressures achievable on the surface of cavitation bubbles, which are the sizes of mechanical stress which is necessary for destruction of polystyrene. That is, the contribution of the bubbles, pulsing from smaller absolute values of the variable pressure in the standing wave is not taken into account. Dispersion of the distribution VDPCE in both options of the reactor was equal to each other and had a size of 


\section{4 .}

A characteristic of variants of the reactor and the results of despergations particles of latex is shown in table 1 . Estimated size of the ratio (10) shows that the acoustic power of the options of reactor is differ in two times, the working volume - in three times, but they produce erosive effects which differ in 2.46 times. The experiment confirms with a $90 \%$ probability, that this difference be within $2.17 \ldots 2.84$. That is, for the chosen quantitative method of testing the correctness of expression (10) the result was quite satisfactory.

\subsection{Predictive function of this method}

Assume that for aforementioned technology is required the device with the greatest possible productivity. In the Russian market is offered to sale ultrasonic generator УЗГМ-10-22 from which can work two ultrasonic heads of type ПМС-5-18 (Company "РЭЛТЕК", Yekaterinburg). It now is the most powerful in Russia a set for two coherent waves in a liquid. Its full acoustic power is $4.4 \mathrm{kw}$. The meanings of the relation (10) for the such reactors with the different sizes to the reactor which is shown in a Fig. 7aб, two-dimensional distributions VDPCE and their dispersion in diametrical sections of working volumes is shown on a Fig. 8. Internal diameter of rectors $100 \mathrm{~mm}$ is a maximum, possible at equality of radiating intensity on all area of ultrasound radiations. The height of reactors in $\mathrm{mm}$ is designated by scale (on the right). At the height equal to odd number of a half wave, radiators operate in a phase, for even number - in opposite phases, what can be made by means of the inverter of phases, which is established on one of two exits of one for them of the generator of frequency.

It can be clearly seen on Fig. 8 that the distribution VDPCE on the flat of symmetry of the reactor is not monotonic function. This occurs because the sound wave, although and transfers the energy that dissipates on the cavitation, but behaves, as a standing wave (its knots and antinodes of pressure are motionless on beam). This means that exist stable in space an areas where in separate parts occurs summation of pressure fluctuations coming mainly with identical or opposite signs. The dispersions of the distributions VDPCE at first-fourth sections in Fig. 8 is smaller than in reactor on Fig. 7a, therefore, these versions of the reactors will be assured no worse the uniformity of treatment of liquids. The highest meanings of the ratios of VDPCE are in $3^{\text {th }}$ and $4^{\text {th }}$ versions. Productivities of these reactors for the processing of latex (since the productivity of laboratory reactor is equal to $7.2 \mathrm{l} / \mathrm{h}$ ) by figures are shown too on Fig. 8. For comparison: for similar by output power the processor UIP-4000 «Hielscher GmbH» with a slightly different design of the reactor has productivity $0.4 \mathrm{~m}^{3} / \mathrm{h}$.

\section{Conclusion}

The above-described mathematical model is only deterministic component of a formalized description of the cavitation process, as equality of frequency of the primary wave and of oscillations of bubbles requires a complete identity of their diameters. But in real liquids there is always distribution of bubbles on their initial diameters. This requires the introduction of a stochastic component of the model. The evaluations basing on model also will be relative and will remain such, pending the availability of methods and tools that can accurately determine the absolute values of the parameters in (10) which was eliminated by dividing each other, because they belong to one liquid. Yet this model has real practical value. The similarity hypothesis of cavitation processes makes it possible to design reactors based on the results of laboratory optimization of these processes. In addition, model has a predictive function, which consists in a simple and rapid evaluation of the effectiveness of the existing acoustic devices to apply them in area of cavitation technology. All it gives her the right to exist.

\section{References}

Ashokkumar M., Rink R., \& Shestakov S. (2011). Hydrodynamic cavitation - an alternative to ultrasonic food processing. Electronic Journal “Technical Acoustics”. [Online] Available: http://www.ejta.org, 2011, 9

Dezhkunov N., et al. (2000). Enhancement of sonoluminescence emission from a multibabble cavitation zone. Ultrasonics Sonochemistry, V7, 1, 19-24. http://dx.doi.org/10.1016/S1350-4177(99)00023-1

Dezhkunov N., Ignatenko P., \& Kotukhov A. (2007). Optimization of the activity of cavitation generated by pulsed ultrasound. Electronic Journal “Technical Acoustics”. [Online] Available: http://www.ejta.org, 2007,16 (in Russian).

Flannigan D., \& Suslik K. (2005). Plasma formation and temperature measurement during single-bubble cavitation. Letters to Nature, 434, 52-55. http://dx.doi.org/10.1038/nature03361

Flynn H. (1982). Patent US 4333796

Floris F. M. (2005). Modeling the Cavitation Free Energy. Journal of Physical Chemistry, 109 (50), B, 24061-24070. http://dx.doi.org/10.1021/jp053457+ 
Gaitan D., Tessien R., \& Hiller R. (2007). Pressure pulses from transient cavitation in high-q resonators. $19^{-t h}$ ICA Congress, Madrid

Jinesh K. B., \& Frenken J. W. M. (2008). Experimental Evidence for Ice Formation at Room Temperature. Physical Review Letters, 101, 036101. http://dx.doi.org/10.1103/PhysRevLett.101.036101

Khavroshkin O., Bystrov V. (2007). Sonoluminescence and Sono-fusion. Applied Physics, 5, 7-14 (in Russian)

Kedrinskiy V. (1975). Dynamics of the cavitation zone at underwater explosion near free surface. J. of Appl. Mechanics and Tech. Physics, 5, 68-78 (in Russian)

Klotz A., \& Hynynen K. (2010). Simulations of the Devin and Zudin modified Rayleigh-Plesset equations to model bubble dynamics in a tube. Electronic Journal "Technical Acoustics", [Online] Available: http://www.ejta.org, 2010, 11.

Simulations of the Devin and Zudin modified Rayleigh-Plesset equations to model bubble dynamics in a tube. Electronic Journal "Technical Acoustics". [Online] Available: http://www.ejta.org, 2010, 11

Knapp R., Daily J., \& Hammitt F. (1970). Cavitation. NY: McGraw Book Company

Krefting D., Mettin R., \& Lauterborn W. (2004). High-speed observation of acoustic cavitation erosion in multibubble systems. Ultrasonics Sonochemistry, 11, 119-123. http://dx.doi.org/10.1016/j.ultsonch.2004.01.006

Lahey R., Taleyarkhan R., \& Nigmatulin R. (2007). Sonofusion technology revisted. Nuclear Engineering and Design, V.237, 1571-1585

Lanin V., Dezhkunov N. \& Tomal V. (2008). Instrumentation for measurement of ultrasonic effects in processes. Technology and design of electronic equipment, 2, 51-55 (in Russian).

Lavrinenko O., Savina E., \& Leonov G. (2007). Modeling mechanical-physical and chemical effects in the collapse of cavitation bubbles. Polzunov Bulletin, 3, 59-63 (in Russian).

Margulis M. (1995a). Sonochemistry and Cavitation.- London: Gordon \& Breach

Margulis M. (1997b). Patent RU 2096934

Margulis M. (2000c). Sonoluminescence. Physics-Uspekhi, 170, 263-287 (in Russian).

Matula T., et al. (1995). Comparison of Multibubble and Single-Bubble Sonoluminescence Spectra. Phys. Rev. Lett., 75, 2602-2605. http://dx.doi.org/10.1103/PhysRevLett.75.2602

Mawson R., \& Knoerzer K. (2007). A brief history of the application of ultrasonics in food processing. $19^{\text {th }} I C A$ Congress, Madrid

Melnikov P., Makarenko V., \& Makarenko M. (2004). Achievement of high temperatures during compression vapor bubble. J. of Appl. Mechanics and Tech. Physics, V45, 4, 13-25 (in Russian).

Mettin R., et al. (1997). Bjerknes forces between small cavitation bubbles in a strong acoustic field. Phys. Rev. 56, 3, 2924-2931. http://dx.doi.org/10.1103/PhysRevE.56.2924

Mettin R., Koch Ph., \& Lauterborn W. (2006). Modeling acoustic cavitation with bubble redistribution $6^{\text {th }}$ International Symposium on Cavitation, Wageningen

Nigmatulin R. (2005). Nano-scale thermonuclear fusion in imploding vapor bubbles. Nuclear Engineering and Design, V.235, 1079-1091. http://dx.doi.org/10.1016/j.nucengdes.2005.02.017

Podobriy G. M., et al. (1969). Theoretical foundations of torpedo weapons. Moscow: Military (in Russian).

Putterman S. et al. (1994). Patent US 5659173

Rogov I., \& Shestakov S. (2004). Epithermal change the thermodynamic equilibrium of water and aqueous solutions: Delusion and Reality. Storage and Processing of Farm Products, 4, 17-20; 10, 9-13 (in Russian).

Rozenberg L. (Eds.) (1968). Physics and technology of high-intensity ultrasound. Moskow: Nauka, (in Russian) Shestakov S. (2001a). The basic technology of cavitation disintegration. Moskow: EVA-Press (in Russian).

Shestakov S. (2007b). Acoustic problem of inertial fusion in caverns Dep. VINITI, 532-B2007 (in Russian).

Shestakov S. (2007c). Patent EP 1810744

Shestakov S. (2008d). Research of an opportunity to strengthen the nonparametric multibubble cavitation. Applied Physics, 6, 18-24 (in Russian). 
Shestakov S. (2009e). Management of hydration the food biopolymers. In V. Panfilov (Eds.). Theoretical Foundations of Food Technology. Moskow: ColosS (in Russian)

Shestakov S. (2010f). Multibubble acoustic cavitation: a mathematical model and physical similarity. Electronic Journal "Technical Acoustics", [Online] Available: http://www.ejta.org, 2010, 14 (in Russian).

Shestakov S., \& Befus A. (2008). The formulation of the criterion of similarity sonochemical reactors processing environments that do not ensure acoustic resonance. Dep. VINITI, 840-B2008 (in Russian).

Shestakov S., \& Krasulya O. (2010). Sonochemical technologies in food industry. Electronic Journal "Technical Acoustics". [Online] Available: http://www.ejta.org, 2010, 10 (in Russian).

Taleyarkhan R. et al. (2002). Evidence for Nuclear Emissions During Acoustic Cavitation. Science, V.295, 1868-1873. http://dx.doi.org/10.1126/science.1067589

Taleyarkhan R. et al. (2004) Additional evidence of nuclear emissions during acoustic cavitation. Physical Review, E. V.69, 036109. http://dx.doi.org/10.1103/PhysRevE.69.036109

Products. Equipment. Ultrasonic technological plants. (2011). [Online] Available: http://www.reltec.biz/en/view_products.php?id=5

Devices. Industrial. UIP-4000. (2011). [Online] Available: http://www.hielscher.com/ultrasonics/i4000_p.htm

Table 1

\begin{tabular}{|l|c|c|c|}
\hline \multirow{2}{*}{ PARAMETERS } & \multirow{2}{*}{ Units } & \multicolumn{2}{c|}{ VALUE } \\
\cline { 3 - 4 } & & & Version a \\
& & 350 & 700 \\
\hline Acoustic power harmonic oscillations & $W$ & 41.4 & 124.2 \\
The volume of the working space of the reactor $V$ & $m l$ & 19.9 & 82.3 \\
The total volume of the cavitation fields $\Sigma v_{i}$ & $m l$ & 0.15 & 0.15 \\
The coefficient of energy dissipation on the cavitation $\eta$ & & -0.88 & - \\
The reflection coefficient of the wave $\theta$ & & $236 \pm 6$ & $143 \pm 7$ \\
The resulting average particle diameter of latex* & $n m$ & 1 & 2.46 \\
Estimated size of the ratio in (10) & & $21 \pm 2$ & $52 \pm 2$ \\
A relative increase in the dispersion of latex & $\%$ & & \\
\hline
\end{tabular}

*with the boundaries of the confidence intervals at $90 \%$ significance level. 


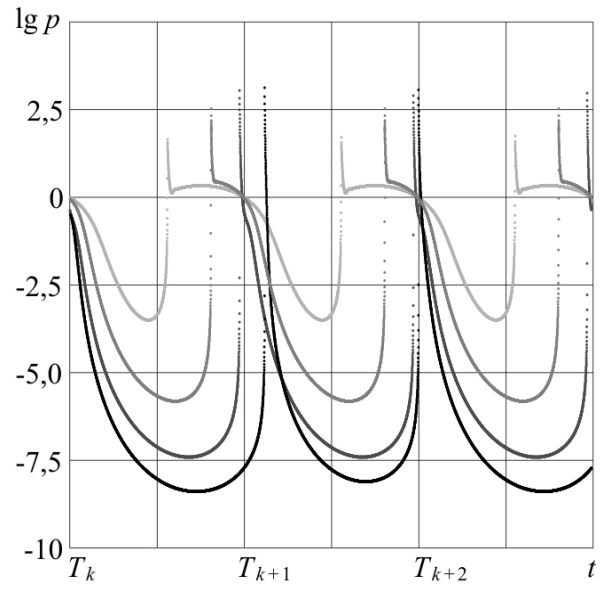

a).

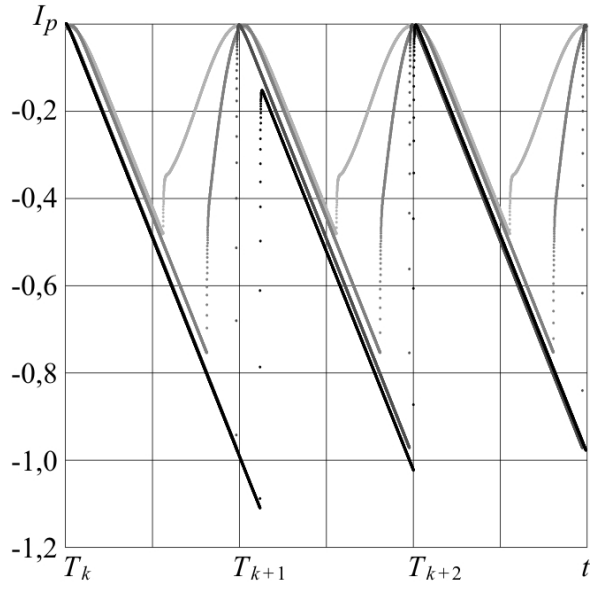

b).

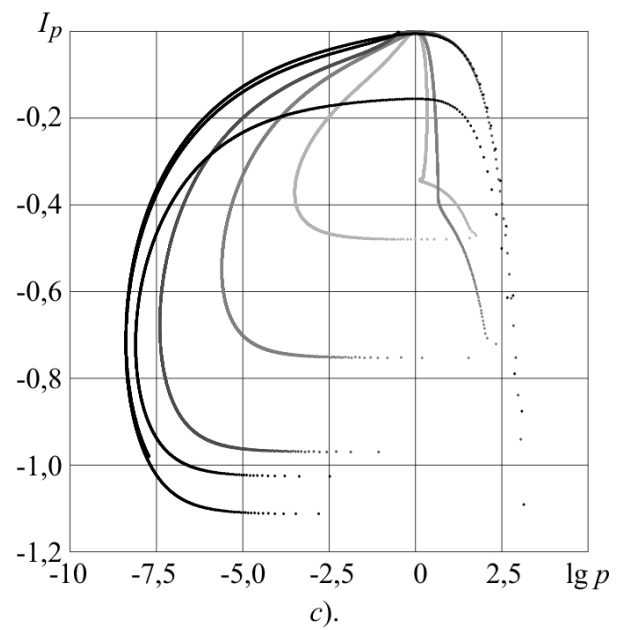

Figure 1. $(a, b, c)$ The graphs of pulsation of a single bubble with initial diameter of $10 \mu m$ in water at amplitudes of sound pressure: $\cdots-1,25$ bar; $\cdots-2,5$ bar; $\cdots-5$ bar; $\cdots-10$ bar with frequency $20 \mathrm{KHz}$ of chemically pure water with atmospheric pressure $P_{h}$ 


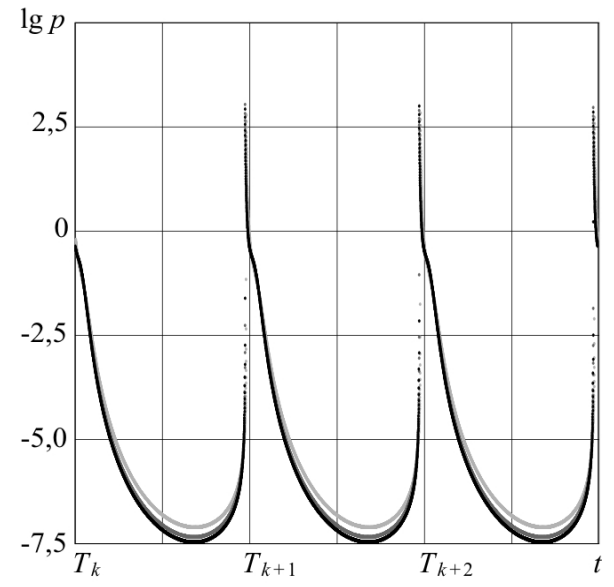

a).

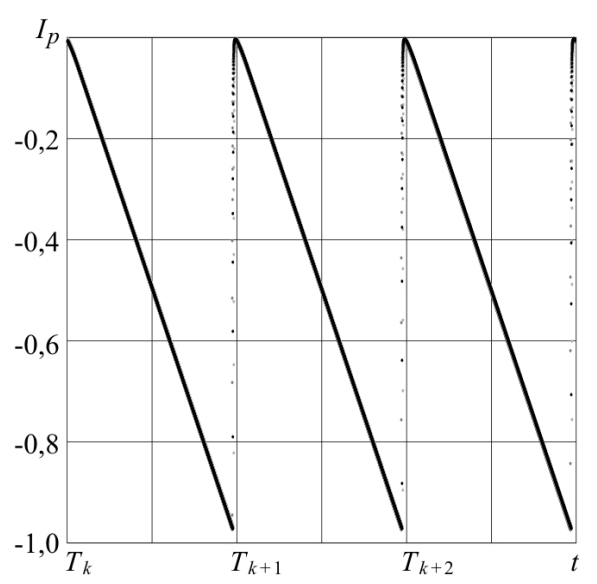

b).

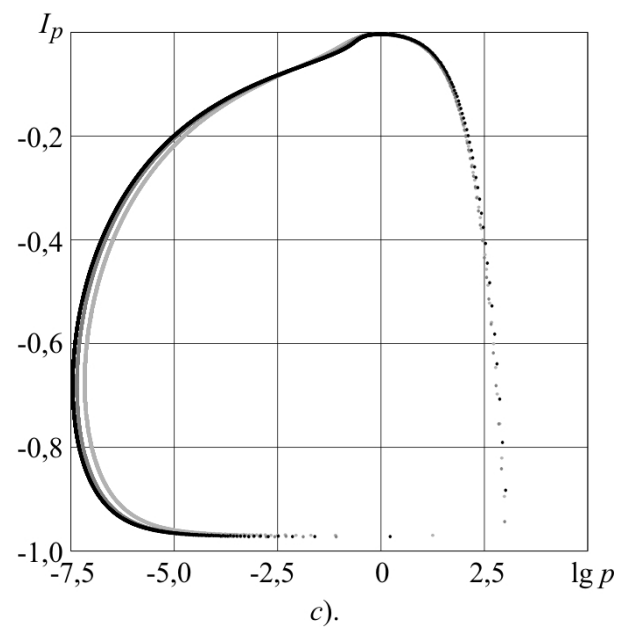

Figure 2. $(a, b, c)$ The graphs of pulsation of a single bubble with initial diameter: $\cdots-1 \mu m ; \cdots-10 \mu m$; •- $100 \mu \mathrm{m}$ with the amplitude of sound pressures in water of $5 \mathrm{bar}$

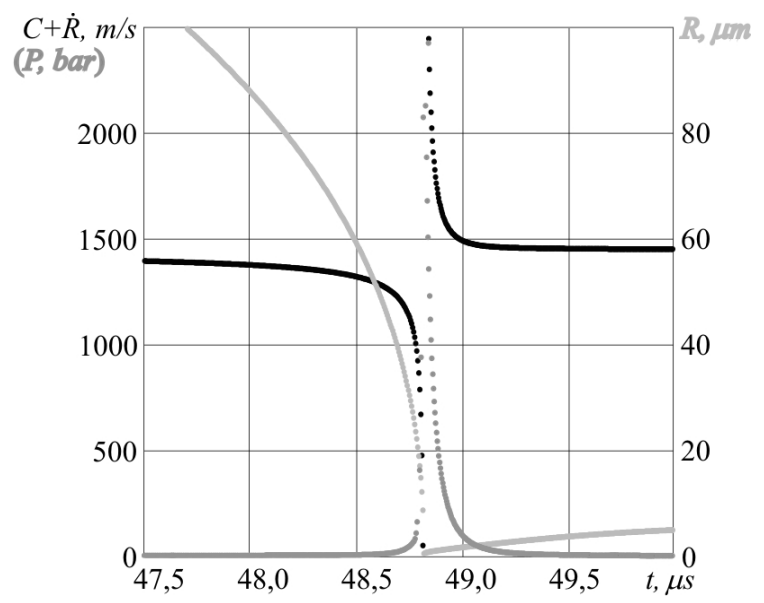

Figure 3. Graphs changes in time of: $\cdots$ - the cavitation bubble radius $R ; \cdots-$ the pressure at him surface $P ; \cdots-$ the speed in water on walls of babble $C+\dot{R}$ in the elastic wave with amplitude of the sound pressure of 5 bar and a frequency of $20 \mathrm{KHz}$ 


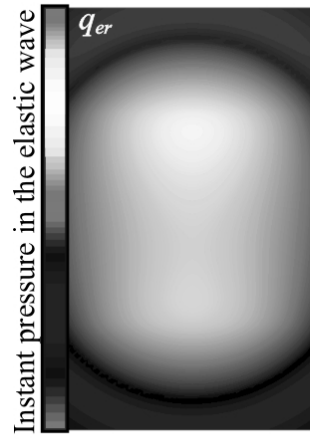

a).

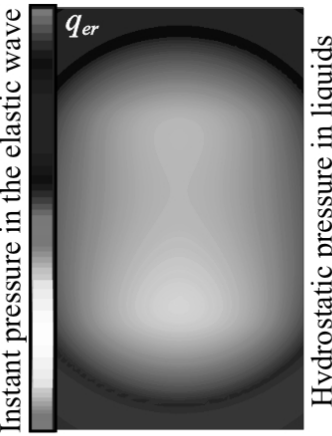

b).

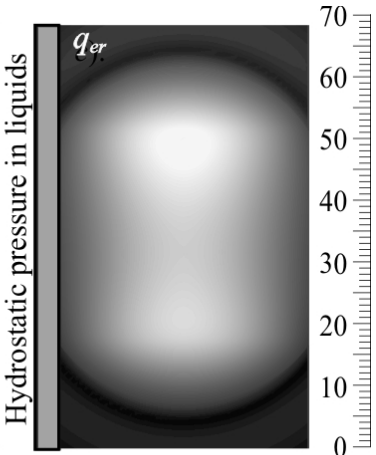

$c)$.

Figure 4. Two-dimensional distribution VDPCE in the water in diametrical section of the reactor with top-mounted emitter $350 \mathrm{~W}$ in the compression phase occurring in: a). the near field of the emitter; $b$ ). a remote area from the emitter and c). VDPCE is averaged over an infinitely long time

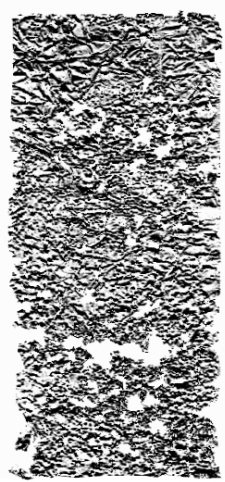

a).

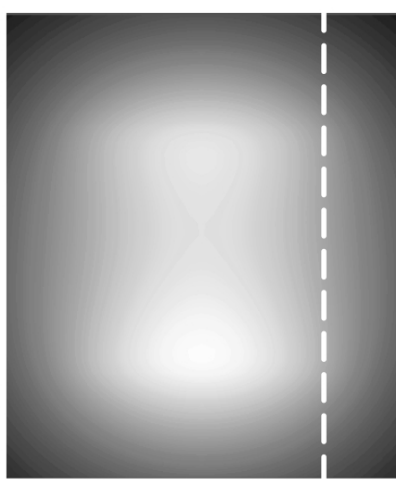

b).

Figure 5. The destruction the aluminum foil from cavitation in the three independents sources of primary waves: a). sample foil with traces of erosion, b). distribution VDPCE relative to the plane of the foil. Numbers show the ratio of VDPCE to the average value in it

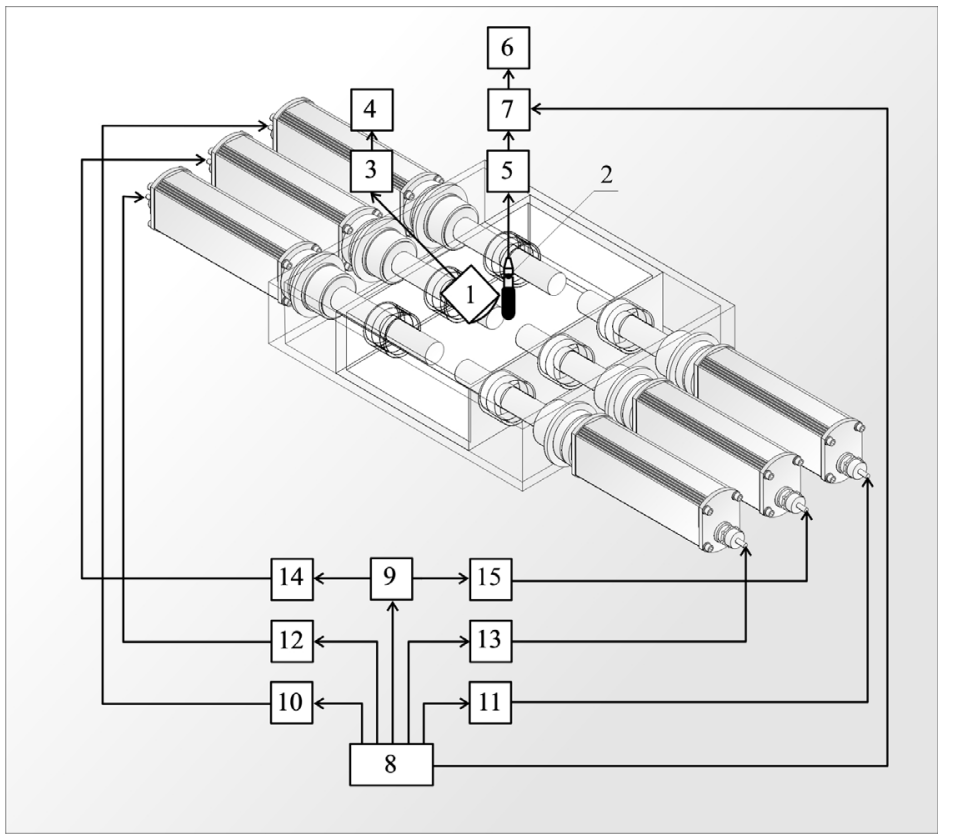

Figure 6. General view and schematic diagram of the experimental reactor to study the effect of nonparametric amplification of cavitation: 1 - photomultiplier tube; 2 - hydrophone; 3, 5- amplifiers; 4, 6 -integrators;

7 - device of strobing; 8 - device synchronization; 9 - delay line; $10 \ldots 15$ - ultrasonic generators 


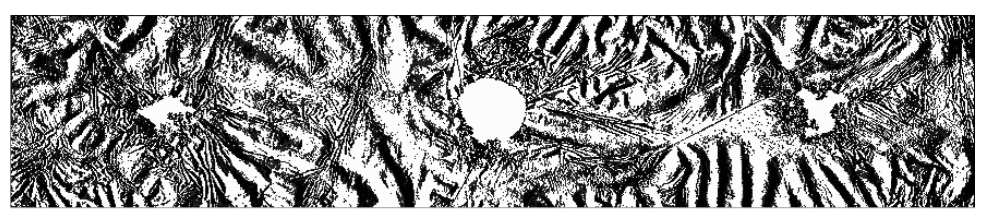

a).

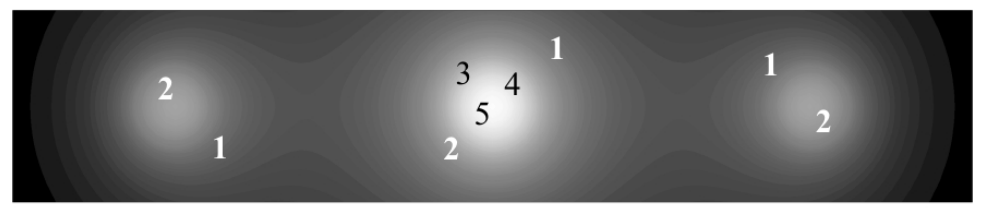

$b)$.

Figure 7. The destruction the aluminum foil from cavitation: a). the erosion of sample foil; $b$ ). distribution VDPCE in diametrical plane of cross-section of the reactor.

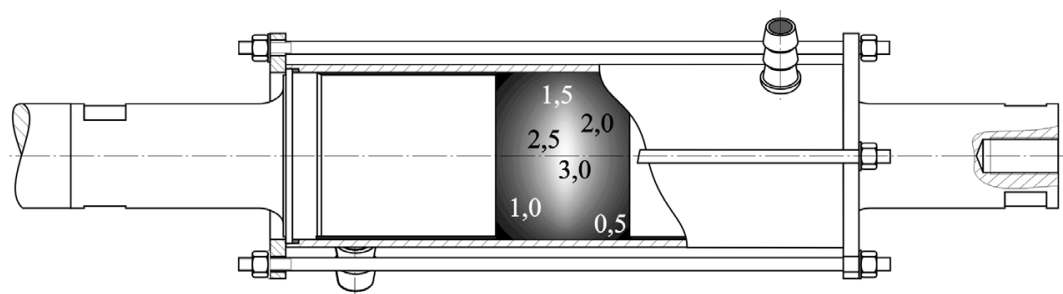

a).

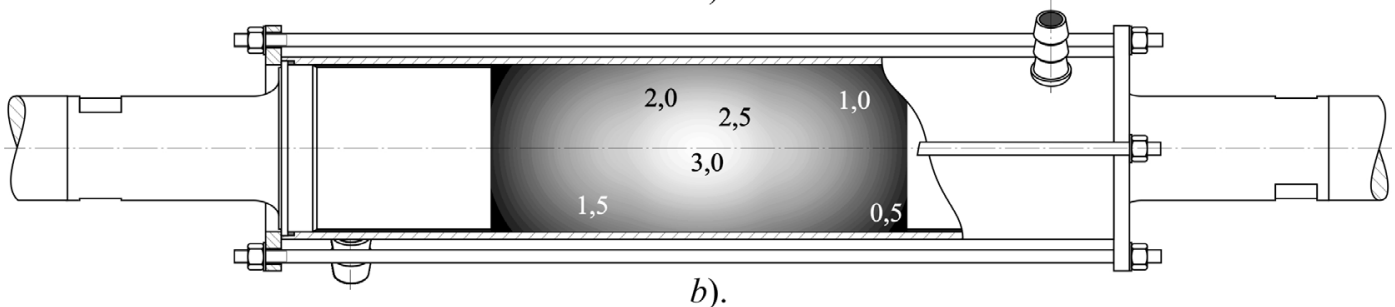

Figure 8. The design of an experimental reactor with a distribution of relative VDPCE in diametrical plane of cross-section of the reactor: a). and b). - versions

Value $(10)=8,8$

Dispersion $=0,30$

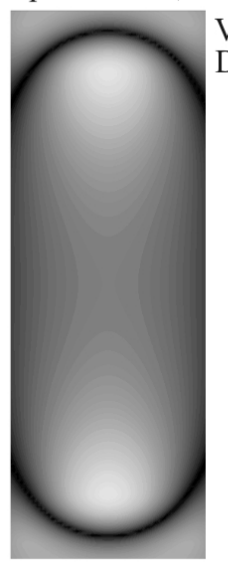

Value $(10)=22,8$
Dispersion $=0,26$
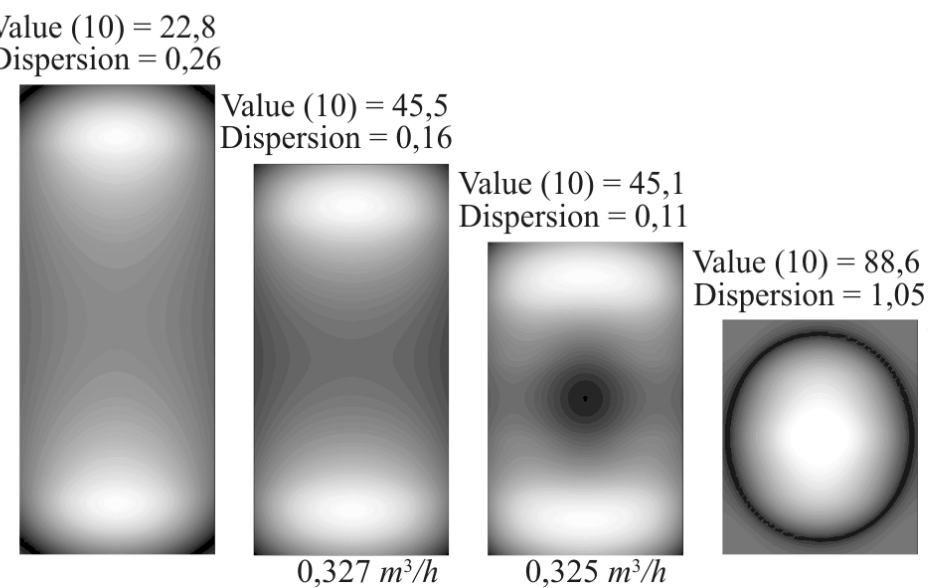

Figure 9. The variants the distributions of VDPCE in diametrical cross-sections of the working volume of cavitation reactor with two radiators type ПMC-5-18 